\title{
Minimizing total tardiness for the order scheduling problem with sequence-dependent setup times using hybrid matheuristics
}

\author{
Massimo Pinto Antoniolia ${ }^{a}$ Carlos Diego Rodriguesa and Bruno de Athayde Prata ${ }^{a^{*}}$
}

\begin{tabular}{l}
${ }^{a}$ Federal University of Ceará, Fortal \\
\hline C H R O N I C L E \\
\hline Article history: \\
Received May 112021 \\
Received in Revised Format \\
June 282021 \\
Accepted October 222021 \\
Available online \\
October, 272021 \\
\hline Keywords: \\
Production Scheduling \\
Matheuristics \\
Mixed-Integer Linear \\
Programming
\end{tabular}

\section{Introduction}

Consumer demand, combined with global competition among firms, has been introducing new production paradigms in a crescent quest for quality, resulting in the allocation of manufacturing of components of specialized goods to several plants or production environments (Fernandez-Viagas \& Framinan, 2015). As a result, in recent years, production scheduling researchers have focused on the assembly scheduling problems, such as the customer order scheduling environment (Framinan et al., 2018). The customer order scheduling problem (COSP) is defined as $n$ orders to be scheduled on $m$ dedicated parallel machines, where each machine can process only one type of operation. The COSP presents several real-world applications, such as paper production, pharmaceutical industry, assembly operations, and in the repair of airplanes and vessels (Magazine and Julien, 1990; Leung et al., 2005b; Roemer, 2006).

Usually, the setup times are embedded in the processing times in the current literature on customer order scheduling (Prata et al., 2021a). Nevertheless, this may not be a realistic assumption in many practical situations since the machines allow for some versatility in handling various items, which typically necessitates some set-up operations. In our proposal, each order presents an associated setup time, which is sequence-dependent, as well as a due date, related to the customer requirements.

Based on recent studies (Framinan et al., 2019, Wu et al., 2021, Prata et al., 2021a, Prata et al., 2021b), we can observe that the variant introduced here is not reported previously. This paper aims at presenting the COSP with sequence-dependent setup times to minimize the total tardiness. We develop a mixed-integer linear programming (MILP) model, as well as explore some problem properties. As a solution procedure, we extend two existing constructive heuristics. Also, we present a matheuristic based on the reduction of the number of decision variables. Finally, we carried out extensive computational experiments with random-generated test instances.

* Corresponding author

E-mail: baprata@ufc.br (B. D. A. Prata)

2022 Growing Science Ltd. doi: $10.5267 / j . i j i e c .2021 .11 .002$ 
The remaining sections of this paper are structured as follows: Section 2 addresses some related approaches, Section 3 describes the problem under study, Section 4 presents the proposed solution approaches, Section 5 summarizes the computational results. Finally, Section 6 addresses the final remarks as well as suggestions for future works.

\section{Literature Review}

Since the considered problem has not been previously reported, we are looking for related approaches. The presented studies are classified based on the notation provided by Framinan et al. (2018). Wagneur and Sriskandarajar (1993) presented the COSP as an open shop scheduling problem in which the overlap of jobs in the available machines is allowed. Furthermore, the order scheduling to minimize the total tardiness performance measure $D P m \rightarrow 0|| \Sigma T$ is classified as NP-hard. A notation for the classification of order scheduling problems is introduced by Leung et al. (2005a). Roemer (2006) addressed a concurrent open shop in which the jobs are scheduled in dedicated parallel machines. Leung et al. (2005b) addressed COSPs with release and due dates. The performance measures $f$ to be minimized are total completion time and total tardiness. Several structural properties and complexity analyses are addressed. In addition, two constructive heuristics, as well as a tabu search metaheuristic, are proposed. Leung et al. (2006) addressed two variants of the COSP two objective functions: the minimization of the maximum lateness and the total number of late orders. A constructive heuristic and an exact method are developed as solution approaches. Lee (2013) addressed an important property for $D P m \rightarrow 0|| \Sigma T$ : the global optimal solution always can be found with fixed permutations in the available machines. In addition, a MILP model, a Branch-and-Bound (B\&B) algorithm, and the Order-Scheduling Modified Due-Date heuristic (OMDD) are presented. Framinan and Perez-Gonzalez (2017) approached the COSP for the minimization of the total completion time. An algorithm using a look-ahead mechanism is developed, which is employed as an initial solution for a Greedy Search Algorithm (GSA) metaheuristic (Karabulut, 2016). Framinan and Perez-Gonzalez (2018) extended this heuristic for total tardiness minimization. Furthermore, the matheuristics JPF and JPO are introduced. The second one is the state-of-the-art algorithm for the $D P m \rightarrow 0|| \Sigma T$. Kung et al. (2018) studied a COSP minimizing the total completion time objective with unequal ready times. As theoretical contributions, several problem properties are addressed, as well as two lower bounds. With respect to solution procedures, four simulated-annealingbased and four genetic-algorithm-based metaheuristics are presented, as well as a B\&B exact method. Riahi et al. (2019) studied the $\mathrm{DPm} \rightarrow 0|| \Sigma \mathrm{C}$ variant. Several priority rules are developed, as well as a new constructive heuristic that considers all feasible positions in the partial permutation for not scheduled orders. A Perturbative Search Algorithm (PSA) metaheuristic is proposed, outperforming the GSA metaheuristics presented by Framinan and Perez-Gonzalez (2017). Lin et al. (2019) studied a COSP minimizing the weighted number of tardy orders. Some theoretical contributions are addressed, such as dominance rules and a lower bound. Furthermore, a B\&B algorithm and four bee-colony-based metaheuristics are presented. $\mathrm{Wu}$ et al. (2019) addressed a COSP considering ready times. Several dominance properties and two lower bounds are presented, which are embedded into a B\&B algorithm. Five constructive heuristics are adapted to this variant. Besides, an Iterated Greedy (IG) algorithm is developed. Wu et al. (2021) addressed the COSP considering due dates and scenariodependent processing times. The performance measure is the minimization of the maximum total tardiness across all scenarios. Four lower bounds, as well as four dominance relations, were proposed. Furthermore, some local search and IG populationbased algorithms were presented. The IG approaches outperformed the OMDD-based heuristics, although they increased the time required for the problem resolution. Prata et al. (2021a) addressed the COSP with explicit setups that depend on the production sequence to minimize the makespan. Two mixed-integer linear programming models are developed for this problem, along with two matheuristics that reduce the number of decision variables. Their so-called Fixed Variable List Algorithm (FVLA) is shown to outperform all other methods under comparison. Prata et al. (2022) addressed the customer order scheduling with sequence-dependent setup times with the total completion time objective. A MILP model and a hybrid discrete differential evolution algorithm are developed. As innovative characteristics, this metaheuristic presents a parameterfree restart operator and two local search procedures based on heuristic dominance properties. Computational experimentation pointed to the superiority of the discrete differential evolution algorithm in comparison with other methods.

\section{Problem description}

The $D P m \rightarrow 0\left|S T_{s d}\right| \Sigma T$ is NP-hard problem since it can be reduced, if all setup times are equal to zero, to the canonical customer order scheduling problem to minimize total completion time, which is known to be an NP-hard problem (Wagneur and Sriskandarajar, 1993). Here we propose a MILP model to find high-quality solutions or even global optimal solutions to the problem under study. Each order $k$ has an associated due date $d_{k}$, a processing time $p_{i k}$ in machine $i$, and a sequencedependent $S_{i k l}$. The problem is to find a sequence of orders where the total tardiness is minimized. Next, we define the tardiness for a given order, as well as the total tardiness minimization. The completion time in machine $i$ for a given order processed in postion $j$ of sequence $\Pi, C_{i, \pi_{j}}(\Pi)$, can be calculated recursively as in Eq. (1):

$$
C_{i, \pi_{j}}(\Pi)=C_{i, \pi_{j-1}}(\Pi)+p_{i, \pi_{j}}+S_{i, \pi_{j-1}, \pi_{j}}, \quad i=1, \ldots, m, \quad j=1, \ldots, n
$$

with $C_{i, \pi_{0}}(\Pi):=0, \forall i$. Therefore, $C_{\pi_{j}}(\Pi)$ is the completion time of the order scheduled in position $j$ is defined as in Eq. (2): 


$$
C_{\pi_{j}}(\Pi)=\max _{1 \leq i \leq m}\left\{C_{i, \pi_{j}}(\Pi)\right\}
$$

In the same way, the tardiness for a given order scheduled in position $j$ can be defined as in Eq. (3):

$$
T_{\pi_{j}}(\Pi)=\max \left\{C_{\pi_{j}}(\Pi)-d_{\pi_{j}} ; 0\right\}
$$

Finally, the total tardiness is calculated as $T(\Pi)=\sum_{j=1}^{n} T_{\pi_{j}}(\Pi)$. Likewise, the summation of the completion times $C(\Pi)$ for a given sequence $\Pi$ can be calculated as $C(\Pi)=\sum_{j=1}^{n} C_{\pi_{j}}(\Pi)$.

The problem notation is presented as follows.

\section{Indices and sets}

Set of machines $I=\{1, \ldots, m\}$;

Set of orders $K=\{1, \ldots, n\}$;

Set of positions $J=\{1, \ldots, n\}$.

\section{Parameters}

$p_{i k}$ : machine-dependent processing time of the order $k$;

$S_{i k l}$ : sequence-dependent setup times.

$d_{k}:$ due date of the order $k$;

$M$ : a sufficiently large number.

\section{Decision variables}

$T_{k}$ : tardiness of order $k$;

$C_{i j}$ : completion time of position $j$ in machine $i$;

$D_{i j}$ : setup time for the order scheduling in the position $j$ of machine $i$.

$x_{i j k}=\left\{\begin{array}{lc}1, & \text { if the order } k \text { is scheduled in the position } j \text { of machine } i . \\ 0, & \text { otherwise }\end{array}\right.$

Thereby, resulting MILP model can be described in the following manner:

$$
\min \sum_{k=1}^{n} T_{k}
$$

subject to:

$$
\begin{aligned}
& \sum_{k=1}^{n} x_{i j k}=1, \quad \forall i \in I, \quad \forall j \in J \\
& \sum_{j=1}^{n} x_{i j k}=1, \quad \forall i \in I, \quad \forall k \in K \\
& S_{i k l}\left(x_{i j-1 k}+x_{i j l}-1\right) \leq D_{i j}, \quad \forall i \in I, \quad \forall j>1, \quad \forall k, l \in K
\end{aligned}
$$




$$
\begin{aligned}
& \sum_{r=1}^{j} D_{i r}+\sum_{k=1}^{n} \sum_{r=1}^{j} p_{i k} \cdot x_{i r k} \leq C_{i j}, \quad \forall i \in I, \quad \forall j \in J \\
& C_{i j}-d_{k}+x_{i j k} \cdot M \leq M+T_{k} \quad \forall i \in I, \forall j \in J, \forall k \in K \\
& x_{i j k} \in\{0,1\}, \quad T_{k} \geq 0, \quad C_{i j} \geq 0, \quad D_{i j} \geq 0, \quad \forall i \in I, \quad \forall k \in K, \quad \forall j \in J
\end{aligned}
$$

Eq. (4) is the objective function to be minimized. Constraints (Eq. 5) and (Eq. 6) are permutation restrictions. Constraint set Eq. (7) determines the setup time of the machine $i$ from order $k$ before order $l$. We consider that all the available machines performed the setup operation previously in the first position. Set of constraints (Eq. 8) computes the completion times of the orders. Constraint set (Eq. (9)) determines the tardiness associated with each customer order. Finally, constraint sets (Eq. (10)) determine the scope of the variables.

We define the big- $M$ value as the summation of the processing and setup times, as in Eq. (11).

$$
M=\sum_{i=1}^{m} \sum_{k=1}^{n} p_{i k}+\sum_{i=1}^{m} \sum_{k=1}^{n} \sum_{l=1}^{n} S_{i k l}
$$

Finally, it should be noticed that for this problem we have to consider the possibility of different permutations for every machine. Contrary to the variant without sequence-dependent setup times, where the optimal solution is obtained applying the same permutation on all the available machines by Lee (2013). Here, we show a counterexample to this fact.

Proposition 1: The optimal solution does not necessarily present the same permutation in all the available machines for the $D P m \rightarrow 0\left|S T_{S D}\right| \sum T_{j}$.

Proof: Considering the following example, as described in Table 1. Also, we consider a due date of 5 units of time (u.t.) for both orders.

\section{Table 1}

Illustrative example.

\begin{tabular}{c|cc}
\multicolumn{3}{|c}{ Processing times } \\
\hline & $O_{1}$ & $O_{2}$ \\
\hline$M_{1}$ & 2 & 1 \\
$M_{2}$ & 3 & 4
\end{tabular}

\begin{tabular}{c|cc}
\multicolumn{3}{|c}{ Setup times-machine 1 } \\
\hline & $O_{1}$ & $O_{2}$ \\
\hline$O_{1}$ & - & 1 \\
$O_{2}$ & 10 & -
\end{tabular}

\begin{tabular}{c|cc}
\multicolumn{3}{c}{ Setup times-machine 2 } \\
\hline & $O_{1}$ & $O_{2}$ \\
\hline$O_{1}$ & - & 2 \\
$O_{2}$ & 1 & -
\end{tabular}

Using the same permutation in all machines, we obtain the results illustrated in Fig. 1, which present a total tardiness of the 4 u.t. and 8 u.t. time units, respectively. If we use distinct permutations in the machines, we can obtain a total tardiness of 3 u.t. time units, as illustrated in Fig. 2. Thus, solutions with distinct permutations in the machines can lead to smaller total tardiness than solutions with fixed permutations in the machines.

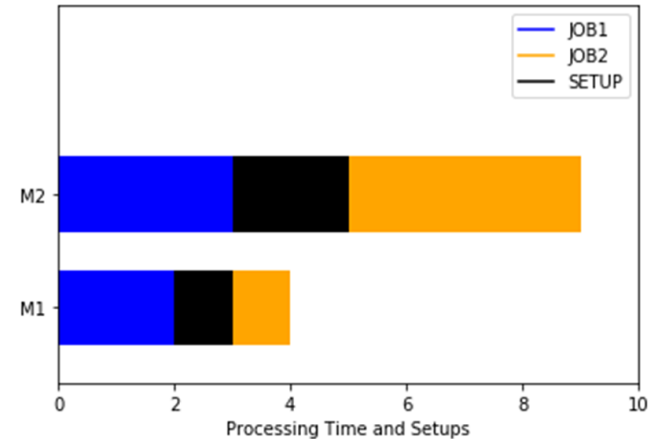

(a)

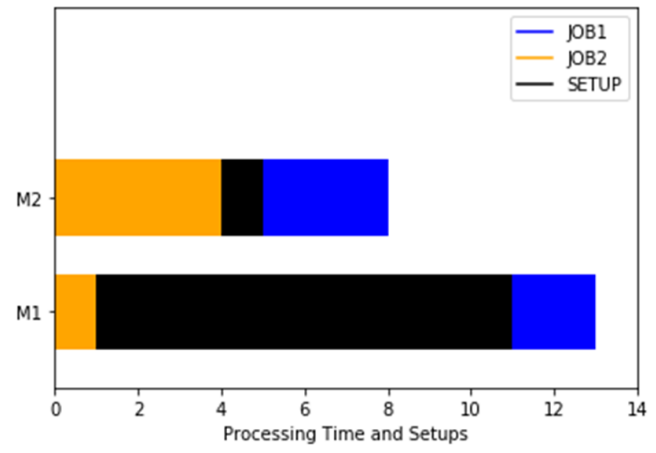

(b)

Fig. 1. Solutions with fixed permutations 


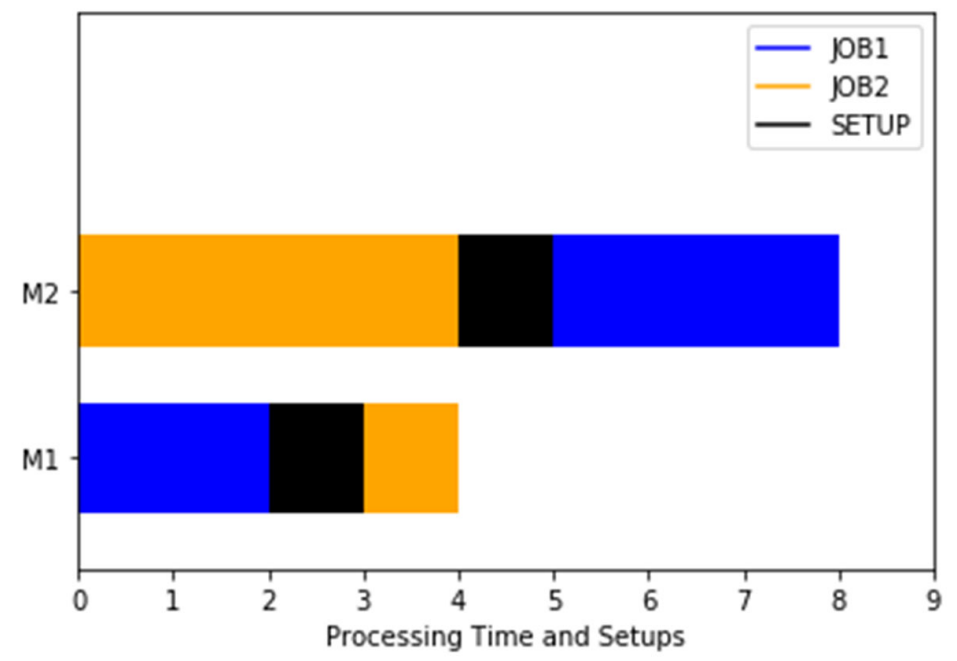

Fig. 2. Solution with distinct permutations.

\section{Proposed solution approaches}

Since the proposed variant is a NP-hard problem, we presented heuristic algorithms to find high-quality solutions within admissible computational time.

\subsection{Constructive heuristics}

The OMDD-S heuristic is an extension of the OMDD proposed by Lee (2013), considering sequence-dependent setup times. Our proposal is described in the following steps.

- $\quad$ Step 0 : Set $\Omega:=(1,2, \ldots, n), \Pi:=\emptyset$ and $t_{i}=0(1 \leq i \leq m)$.

- $\quad$ Step 1: Choose a given order in $\Omega$ using the index $\alpha_{j}$ :

$$
\alpha_{j}=\max \left\{\max _{1 \leq i \leq m}\left\{t_{i}+S_{i r j}+p_{i j}\right\}-\max _{1 \leq i \leq m}\left\{t_{i}\right\} ; d_{j}-\max _{1 \leq i \leq m}\left\{t_{i}\right\}\right\} .
$$

- $\quad$ Step 2: Delete a given order from $\Omega$, including it at the end of $\Pi$.

- $\quad$ Step 3: Refresh $t_{i}$. If $|\Omega|=n-1$, then $t_{i}:=t_{i}+p_{i r}$; otherwise, $t_{i}:=t_{i}+S_{i w r}+p_{i r}$, where $w$ is the last but one elemetn from $\Pi$.

- $\quad$ Step 4: While $\Omega$ is not empty, return to Step 1 .

Also, we present the FP-S heuristic, as an extension of the FP algorithm (Framinan and Perez-Gonzalez, 2018), including the sequence-dependent setup times. The above-mentioned algorithm builds a permutation $\Pi:=\left(\pi_{1}, \ldots, \pi_{n}\right)$ from a set of unscheduled orders $\mathcal{U}$. Initially, $\mathcal{U}$ has all the orders since none of the orders was scheduled. In iteration $\mathcal{K}$, each order in $\mathcal{U}$ is chosen to be inserted in the last position of $\Pi$. Aiming to choose a given order from the candidate list, considering $\omega_{l} \in \mathcal{U}$ with a due date $d_{l}$, a partial permutation $S_{l}$ is builded inserting $\omega_{l}$ in the last position of $\Pi$, i.e. $\Pi:=\left(\pi_{1}, \ldots, \pi_{k-1}, \omega_{l}\right)$.

Therefore, the tardiness indicator $\eta_{l}$ for the candidate $l$ is calculated as in Equation (Eq.13):

$$
\eta_{l}=\max \left\{C\left(S_{l}\right)-d_{l} ; 0\right\}+T S_{l}
$$

In which the first term is the portion of the total tardiness if $I$ is selected, in which $C S_{I}$ is the partial completion time of the permutation $S_{l}$ and $d_{l}$ is the associated due date. The second term $T S_{l}$ estimates the parcel for the total tardiness of the remainder orders. Fig. 3 illustrates the FP-S constructive heuristic. 
We adopt as a permutation in which all the orders are included before the procedure. For each iteration, we calculate $T S_{I}$ considering the remnant orders are allocated after the orders previously selected. We consider the sequence returned by the Earliest Due Date (EDD) heuristic. This algorithm is a well-known priority rule to solve a scheduling problem with due dates. We select a given order, append it at the end of the partial permutation, and remove it. As the remnant orders were already selected, they are not calculated again in the next iteration. Our FP-S heuristic is described as follows.

- Step 0: Determine an initial permutation $\Omega$ using the EDD dispatch rule.

- Step 1: For each position $j$ of the sequence, calculate the indicator for each unscheduled order.

- Step 2: For each $\omega_{l} \in \Omega$, estimate the completion times of $\omega_{l}$ for each available machine.

- Step 3: Determine the first term of indicator $\eta_{l}$, using Equation (Eq. 13). Next, determine the second term of indicator $\eta_{l}$.

- Step 4: Choose the order presenting the smaller value of $\eta_{l}$. Insert $\omega_{l}$ at the end of permutation $\Pi$. Update the list $\Omega$.

- Step 5: Update the completion times for each machine.

\subsection{Matheuristics}

\subsubsection{SPAM}

As in the problem under study, each machine can receive a distinct sequence we face a problem with a non-permutational encoding. We can observe that this encoding does not necessarily lead to better solutions because the search space can increase substantially. Fernandez-Viagas et al. (2019) demonstrate that permutational encodings can provide better results than nonpermutational encodings in other production scheduling problems. This is because a solution procedure can evaluate a higher number of solutions with a permutational encoding, for a given time limit.

An intuitive procedure to solve the problem under study is to consider a fixed permutation in all machines. In this way, we can replace the decision variable $x_{i j k}$ in the model defined in Equations (Eq.4) - (Eq.10) by a decision variable $x_{k j}$ equals to 1 if the order $\mathrm{k}$ is processed in position j, 0 otherwise. The Same Permutation in All Machines (SPAM) matheuristic reduces the number of binary decision variables from $m n^{2}$ to $n^{2}$. We can observe that the optimal solution returned by the SPAM matheuristic is not necessarily the optimal solution to the original problem.

\subsubsection{A hybrid matheuristic}

We also propose a hybrid matheuristic based on the JPO algorithm proposed by Framinan and Perez-Gonzalez (2018), denominated Job-Position Oscillation with the Same Permutation in All Machines (SPAM-JPO). We consider only solutions with a fixed permutation, restricting that a given order could be replaced $\delta$ positions at most in the sequence.

Let a permutation $\Pi$ with all orders $k(k=1, \ldots, K)$, we can obtain the position $w_{k}(\Pi)$ of order $k$. Therefore, a set of feasible positions for the order $k$ is determined so that the order $\mathrm{k}$ can be replaced with a maximal number of $\delta$ positions backward or forward. The value $\delta$ is a required input of the matheuristic. The set of possible positions for the order $k$ is given by $k$ é $\mathcal{P}_{k}(\Pi, \delta)=\left\{w_{k}(\Pi, \delta), w_{k}(\Pi, \delta)+1, \ldots, \bar{w}_{k}(\Pi, \delta)\right\}, \quad$ where $\quad w_{k}(\Pi, \delta)=\max \left\{0 ; w_{k}(\Pi)-\delta\right\} \quad$ and $\quad \bar{w}_{k}(\Pi, \delta)=$ $\min \left\{n ; w_{k}(\Pi)^{-}+\delta\right\}$. Thereby, for each order $k$ the decision variables $x_{k j}$ are not significant for $j \in \mathcal{P}_{k}(\Pi, \delta)$. Similarly, the set of orders that can be placed in a position $j$ can be stated as $\mathcal{O}_{j}(\Pi, \delta)=\left\{k: j \in \mathcal{P}_{k}(\Pi, \delta)\right\}$. The hybrid matheuristic SPAMJPO is expressed as follows.

$$
\min \sum_{j=1}^{n} T_{j}
$$

subject to:

$$
\sum_{k \in \mathcal{O}_{j}(\Pi, \delta)} x_{k j}=1, \quad \forall j \in J
$$

$$
\sum_{j \in \mathcal{P}_{k}(\Pi, \delta)} x_{k j}=1, \quad \forall k \in K
$$




$$
\begin{aligned}
& S_{i k l}\left(x_{k j-1}+x_{l j}-1\right) \leq D_{i j}, \quad \forall i \in I, \quad \forall j>1, \quad \forall k, l \in K \\
& \sum_{r=w_{k}(\Pi, \delta)}^{\min \left\{j: \bar{w}_{k}(\Pi, \delta)\right\}} D_{i r}+\sum_{k \in O_{j}(\Pi, \delta)} \sum_{\substack{r=w_{k}(\Pi, \delta) \\
--}}^{\min \left\{j: \bar{w}_{k}(\Pi, \delta)\right\}} p_{i k} \cdot x_{k r} \leq C_{j}, \quad \forall i \in I, \quad \forall j \in J \\
& C_{j}-\sum_{k \in O_{j}(\Pi, \delta)} d_{k} \cdot x_{k j} \leq T_{j}, \quad \forall j \in J \\
& x_{k j} \in\{0,1\}, \quad T_{j} \geq 0, \quad C_{j} \geq 0, \quad D_{i j} \geq 0, \quad \forall i \in I, \quad \forall k \in K, \quad \forall j \in J, \\
& j \in \mathcal{P}_{k}(\Pi, j)
\end{aligned}
$$

Eq. (19) is the total tardiness minimization. Constraint sets Eq. (15) and Eq. (16) impose that the problem solution is a fixed permutation. Constraint set Eq. (17) calculates the setup time for the order scheduled in the position $j$ of the machine $i$. Constraint set Eq. (18) calculates the completion time of each order processed in position $j$. Constraint set Eq. (19) defines the tardiness for of each order processed in position $j$. Lastly, constraint sets Eq. (20) express the domain of the decision variables.

Our SPAM-JPO matheuristic can be summarized as follows. Taking an initial permutation into account, determine a corresponding solution with the positional decision variables $x_{k j}$. While a general time limit $t_{\text {limit }}$ is not exceeded, solve the MILP model defined by Eqs. (14-20), using a permutation $\Pi$, an oscillation parameter $\delta$, and a secondary time limit $t_{w}$. Update the solution found as well as the parameter $\delta$. Finally, return the best solution found.

\section{Computational results}

\subsection{Experimental design}

Aiming to perform a fair comparison between the evaluated methods, we developed a testbed similar to the test problems proposed by Lee (2013). However, in our test instances, we consider the generation of sequence-dependent setup times. We generate two data sets: the small-sized test instances with $m \in\{3,5,9\}, n \in\{8,12,16\}$, and the large-sized test instances with $m \in\{2,5,8\}$ and $n \in\{10,20,30,40\}$. Since the problem under study was not reported yet, we randomly generated the evaluated test instances, based on the related instances previously presented. The testbed is composed of test instances with $m \in\{2,5,8\}$ and $n \in\{10,20,30,40\}$. The processing times follow a uniform distribution $U[1,100]$. The sequence-dependent setup times follow three uniform distributions $\{U[1,25], U[26,75], U[76,125]\}$. The due date $d_{k}$ follows a uniform distribution, where:

- $\quad P$ is the summation of processing and setup times divided by the number of available machines. Aiming to generate tight due dates, we multiply this expression by a factor $\mu$.

$$
P=\left[\frac{\sum_{i=1}^{m} \sum_{k=1}^{n} p_{i k}+\sum_{i=1}^{m} \sum_{k=1}^{n} \sum_{l=1}^{n} S_{i k l}}{m}\right] \cdot \mu,
$$

- $R D D$ is the interval for due dates: $R D D:\{0.2,0.5,0.8\}$;

- $T F$ is the tardiness factor for the due dates: $T F:\{0.2,0.5,0.8\}$

For each combination of parameters were generated 10 test instances, totalizing 3240 instances. The methods under comparison are listed below.

- EDD heuristic (a well-know algorithm for problems with due dates);

- OMDD heuristic proposed by Lee (2013);

- FP heuristic proposed by Framinan and Perez-Gonzalez (2018);

- JPO matheuristic by Framinan and Perez-Gonzalez (2018);

- The proposed OMDD-S heuristic; 
- The proposed FP-S heuristic;

- The proposed SPAM matheuristic;

- The proposed hybrid matheuristic SPAM-JPO;

- $\quad$ The proposed MILP model.

We can observe that the warm start of the JPO and SPAM-JPO matheuristics was the OMDD-S. Framinan and Perez-Gonzalez (2018) used the FP heuristic as the warm start. We decided to use the OMDD-S as the initial solution, taking into consideration preliminarily computational experiments.

The methods under comparison were evaluated considering four indicators:

- $\quad$ Relative Deviation Index $(R D I)$ indicator as a performance measure, which is a standard indicator for scheduling problems with due-date objectives. Let $H$ be a set of methods, the $R D I$ found for the method $s \in H$ when tested in instance $t$ is calculated as in Eq. (21).

$R D I_{s t}=\left\{\begin{array}{cc}0, & \text { if } \min _{h \in H} T_{h t}=\max _{h \in H} T_{h t}, \\ \frac{T_{s t}-\min _{h \in H} T_{h t}}{\max _{h \in H} T_{h t}-\min _{h \in H} T_{h t}} \cdot 100, & \text { otherwise. }\end{array}\right.$

where $T_{s t}$ is the tardiness value returned by method $s$ in instance $t$. In our study, $\min _{h \in H} T_{h t}$ the best solution found among the methods under comparison.

- $\quad$ SR (success rate). This indicator is determined as the number of instances in which a given solution procedure return the better solution divided by the number of evaluated test instances Eq. (21):

$$
S R=\frac{n_{B E S T}}{n_{I N S T}} \times 100
$$

where $n_{B E S T}$ is the number of test problems where the solution procedure returned the best solution and $n_{I N S T}$ is the number of evaluated test problems.

- Optimality (Opt) evaluates if the solution obtained by the method under comparison in each test instance is the global optimal solution.

- $\quad$ computational time, expressed in seconds.

For the small-sized and large-sized test problems, we generated 10 instances for each combination of parameters, totalizing 2430 and 3240 instances, respectively. The proposed test instances are available here: https://www.researchgate.net/publication/352289324_Evaluated_Instances. The methods under comparison were implemented with Python 3.7. Furthermore, the MILP model and the SPAM matheuristic were solved with IBM ILOG CPLEX version 12.8.0. For all the evaluated methods, we consider a time limit $t_{\text {limit }}=600 \mathrm{~s}$. All the algorithms were executed in an Intel Core i5-3470 with $3.20 \mathrm{GHz}$ and 8 GB RAM.

\subsection{Results and discussion}

\subsubsection{Small-sized test instances}

Tables 2 and 3 illustrate the computational results for different $m$ and $n$ values, considering the average RDI values, the standard deviations, the percentage of optimal solutions found, and the average computational times. We can observe that in the first set of instances $(m=3$ and $n=8)$, the MILP model presented better results. However, with the increase of the size of the problems, the matheuristic SPAM returned better results. Based on Table 4, we can emphasize that the MILP model returned the largest number of optimal solutions for the small-sized test instances. 
Table 2

Small-sized test instances: RDI values for $m$ and $n$ (part 1)

\begin{tabular}{cccccccccccccc}
\hline$m$ & $n$ & & EDD & & & \multicolumn{3}{c}{ FP } & \multicolumn{3}{c}{ FP-S } \\
& & Average & St. Dv. & Time & Average & St. Dv. & Time & Average & St. Dv. & Time & Average & St. Dv. & Time \\
\hline 3 & 8 & 95.5924 & 10.7901 & 0.0000 & 72.7618 & 26.1200 & 0.0010 & 45.0979 & 24.0381 & 0.0014 & 69.9282 & 25.4258 & 0.0010 \\
& 12 & 97.9772 & 6.9836 & 0.0001 & 75.9761 & 25.5007 & 0.0030 & 48.9008 & 22.8246 & 0.0045 & 72.9033 & 27.9774 & 0.0030 \\
& 16 & 92.1670 & 24.7728 & 0.0001 & 73.274 & 30.1649 & 0.0066 & 47.3200 & 26.5247 & 0.0103 & 70.0654 & 31.4608 & 0.0069 \\
\hline 5 & 8 & 93.2917 & 14.4990 & 0.0000 & 68.9482 & 25.3962 & 0.0013 & 42.6921 & 23.2525 & 0.002 & 63.9066 & 27.7710 & 0.0013 \\
& 12 & 95.1717 & 12.1162 & 0.0001 & 72.4786 & 24.0260 & 0.0038 & 46.8317 & 23.7087 & 0.0062 & 70.3361 & 27.2906 & 0.0039 \\
& 16 & 88.7469 & 26.0195 & 0.0001 & 69.2486 & 28.9762 & 0.0087 & 48.4049 & 27.7139 & 0.0144 & 67.2913 & 30.6391 & 0.0090 \\
\hline 9 & 8 & 91.3742 & 16.2296 & 0.0000 & 68.3929 & 25.7720 & 0.0019 & 42.1489 & 22.6573 & 0.0031 & 60.788 & 26.8431 & 0.0019 \\
& 12 & 90.7482 & 15.9556 & 0.0001 & 68.6689 & 23.9219 & 0.0056 & 44.3806 & 22.2601 & 0.0098 & 64.7505 & 25.2717 & 0.0058 \\
& 16 & 80.2203 & 29.7194 & 0.0001 & 60.9753 & 29.9828 & 0.0127 & 40.2145 & 25.8252 & 0.0226 & 57.2266 & 31.6399 & 0.0131 \\
\hline \multicolumn{2}{c}{ Total } & 91.6988 & 19.4780 & 0.0001 & 70.0805 & 27.0107 & 0.005 & 45.1101 & 24.5031 & 0.0083 & 66.3551 & 28.7118 & 0.0051 \\
\hline
\end{tabular}

Table 3

Small-sized test instances: RDI values for $m$ and $n$ (part 2)

\begin{tabular}{|c|c|c|c|c|c|c|c|c|c|c|}
\hline \multirow[t]{2}{*}{$m$} & \multirow[t]{2}{*}{$n$} & \multicolumn{3}{|c|}{ OMDD-S } & \multicolumn{3}{|c|}{ SPAM } & \multicolumn{3}{|c|}{ MILP } \\
\hline & & Average & St. Dv. & Time & Average & St. Dv. & Time & Average & St. Dv. & Time \\
\hline \multirow[t]{3}{*}{3} & 8 & 39.6805 & 22.7597 & 0.0003 & 5.9073 & 8.6510 & 5.2375 & 2.8183 & 7.1515 & 334.8832 \\
\hline & 12 & 39.6883 & 23.0271 & 0.0007 & 2.6774 & 6.7317 & 438.3857 & 18.1819 & 20.9791 & 601.7716 \\
\hline & 16 & 41.2252 & 26.7577 & 0.0011 & 2.2289 & 6.2924 & 542.1021 & 23.2568 & 25.6060 & 540.4455 \\
\hline \multirow[t]{3}{*}{5} & 8 & 34.9788 & 21.1950 & 0.0005 & 2.2768 & 4.9861 & 9.7212 & 23.7074 & 28.8696 & 550.4171 \\
\hline & 12 & 38.3124 & 23.1388 & 0.0009 & 1.0847 & 5.5263 & 479.4585 & 46.7985 & 30.8030 & 600.1471 \\
\hline & 16 & 36.8398 & 25.4770 & 0.0015 & 1.3108 & 5.0498 & 555.001 & 49.9690 & 33.9772 & 549.4129 \\
\hline \multirow[t]{3}{*}{9} & 8 & 32.1479 & 20.2378 & 0.0006 & 0.0859 & 0.7310 & 19.4298 & 56.5587 & 30.3852 & 600.2293 \\
\hline & 12 & 35.4173 & 21.4594 & 0.0014 & 0.5604 & 4.0074 & 555.9576 & 68.1536 & 29.6034 & 608.7139 \\
\hline & 16 & 29.3475 & 24.8520 & 0.0022 & 1.5982 & 6.1035 & 565.6225 & 67.5235 & 35.8523 & 582.6817 \\
\hline \multicolumn{2}{|c|}{ Total } & 36.4042 & 23.5445 & 0.0010 & 1.9700 & 5.9310 & 352.324 & 39.6631 & 35.7772 & 552.078 \\
\hline
\end{tabular}

Table 4

Small-sized test instances: optimality percentage for $m$ and $n$

\begin{tabular}{|c|c|c|c|c|c|c|c|c|}
\hline$m$ & $n$ & EDD & FP & FP-S & OMDD & OMDD-S & SPAM & MILP \\
\hline \multirow[t]{3}{*}{3} & 8 & $0.00 \%$ & $0.00 \%$ & $1.11 \%$ & $0.00 \%$ & $1.11 \%$ & $17.78 \%$ & $61.11 \%$ \\
\hline & 12 & $0.00 \%$ & $0.00 \%$ & $0.00 \%$ & $0.00 \%$ & $0.00 \%$ & $0.00 \%$ & $1.11 \%$ \\
\hline & 16 & $6.30 \%$ & $6.30 \%$ & $6.30 \%$ & $6.30 \%$ & $6.30 \%$ & $10.37 \%$ & $12.22 \%$ \\
\hline \multirow[t]{3}{*}{5} & 8 & $0.00 \%$ & $0.37 \%$ & $0.00 \%$ & $0.00 \%$ & $0.00 \%$ & $4.07 \%$ & $17.04 \%$ \\
\hline & 12 & $0.00 \%$ & $0.00 \%$ & $0.00 \%$ & $0.00 \%$ & $0.00 \%$ & $0.00 \%$ & $0.74 \%$ \\
\hline & 16 & $5.93 \%$ & $5.93 \%$ & $5.93 \%$ & $5.93 \%$ & $5.93 \%$ & $9.26 \%$ & $11.48 \%$ \\
\hline \multirow[t]{4}{*}{9} & 8 & $0.00 \%$ & $0.00 \%$ & $0.00 \%$ & $0.00 \%$ & $0.00 \%$ & $0.37 \%$ & $1.11 \%$ \\
\hline & 12 & $0.00 \%$ & $0.00 \%$ & $0.00 \%$ & $0.00 \%$ & $0.00 \%$ & $0.00 \%$ & $0.00 \%$ \\
\hline & 16 & $5.56 \%$ & $5.56 \%$ & $5.56 \%$ & $5.56 \%$ & $5.56 \%$ & $7.04 \%$ & $7.78 \%$ \\
\hline & & $1.98 \%$ & $2.02 \%$ & $2.10 \%$ & 0.0198 & $2.10 \%$ & $5.43 \%$ & $12.51 \%$ \\
\hline
\end{tabular}

Tables 5 and 6 present the same aggregated results analyzed by the $T F$ and $R D D$ values. We can observe that the SPAM matheuristic returned the better results, expressed in terms of the average and standard deviation values. Analyzing Table 7 , we can also observe that the MILP model obtained the largest percentual of optimality.

Table 5

Small-sized test instances: RDI values for $T F$ and $R D D$ (part 1)

\begin{tabular}{|c|c|c|c|c|c|c|c|c|c|c|c|c|c|}
\hline \multirow[t]{2}{*}{$T F$} & \multirow[t]{2}{*}{$R D D$} & \multicolumn{3}{|c|}{ EDD } & \multicolumn{3}{|c|}{ FP } & \multicolumn{3}{|c|}{ FP-S } & \multicolumn{3}{|c|}{ OMDD } \\
\hline & & Average & St. Dv. & Time & Average & St. Dv. & Time & Average & St. Dv. & Time & Average & St. Dv. & Time \\
\hline \multirow[t]{3}{*}{0.2} & 0.2 & 97.2370 & 8.8203 & 0.0001 & 78.5159 & 26.0079 & 0.0049 & 55.4418 & 27.1227 & 0.0083 & 77.8198 & 27.5458 & 0.0051 \\
\hline & 0.5 & 88.6677 & 27.4747 & 0.0001 & 70.8692 & 31.8983 & 0.0050 & 49.3033 & 28.6754 & 0.0082 & 68.6830 & 32.7437 & 0.0051 \\
\hline & 0.8 & 85.1766 & 31.4354 & 0.0001 & 69.8468 & 33.359 & 0.0049 & 49.5453 & 30.5102 & 0.0082 & 65.3195 & 34.7565 & 0.0051 \\
\hline \multirow[t]{3}{*}{0.5} & 0.2 & 94.2726 & 12.7031 & 0.0001 & 72.1748 & 26.3138 & 0.0050 & 46.3215 & 22.3224 & 0.0082 & 72.3025 & 25.9000 & 0.0051 \\
\hline & 0.5 & 92.1407 & 15.6173 & 0.0000 & 72.5721 & 25.2546 & 0.0049 & 44.8446 & 21.2566 & 0.0083 & 67.2162 & 28.1337 & 0.0051 \\
\hline & 0.8 & 89.5994 & 20.0316 & 0.0001 & 70.2081 & 26.2634 & 0.0050 & 42.7151 & 24.0551 & 0.0083 & 65.9920 & 29.0104 & 0.0051 \\
\hline \multirow[t]{3}{*}{0.8} & 0.2 & 94.3325 & 13.0639 & 0.0001 & 66.6091 & 23.0282 & 0.0050 & 42.7166 & 20.5596 & 0.0082 & 58.6990 & 24.3036 & 0.0051 \\
\hline & 0.5 & 92.2061 & 16.5154 & 0.0001 & 63.613 & 23.5619 & 0.0050 & 37.7572 & 18.4806 & 0.0082 & 59.3584 & 24.4476 & 0.0051 \\
\hline & 0.8 & 91.6570 & 15.9317 & 0.0001 & 66.3153 & 22.9051 & 0.0050 & 37.3459 & 19.1649 & 0.0083 & 61.8058 & 24.6797 & 0.0051 \\
\hline \multicolumn{2}{|c|}{ Total } & 91.6988 & 19.4780 & 0.0001 & 70.0805 & 27.0107 & 0.0050 & 45.1101 & 24.5031 & 0.0083 & 66.3551 & 28.7118 & 0.0051 \\
\hline
\end{tabular}


Table 6

Small-sized test instances: RDI values for $T F$ and $R D D$ (part 2)

\begin{tabular}{|c|c|c|c|c|c|c|c|c|c|c|}
\hline \multirow[t]{2}{*}{$T F$} & \multirow[t]{2}{*}{$R D D$} & \multicolumn{3}{|c|}{ OMDD-S } & \multicolumn{3}{|c|}{ SPAM } & \multicolumn{3}{|c|}{ MILP } \\
\hline & & Average & St. Dv. & Time & Average & St. Dv. & Time & Average & St. Dv. & Time \\
\hline \multirow[t]{3}{*}{0.2} & 0.2 & 51.5566 & 24.1698 & 0.0010 & 4.3980 & 8.6981 & 373.9367 & 22.5721 & 29.3454 & 541.7492 \\
\hline & 0.5 & 44.0141 & 27.3227 & 0.0010 & 3.3462 & 7.2883 & 306.9237 & 27.1313 & 34.3152 & 494.8373 \\
\hline & 0.8 & 42.4777 & 30.1257 & 0.0010 & 4.0201 & 9.2227 & 297.3631 & 27.2049 & 34.7209 & 482.5090 \\
\hline \multirow[t]{3}{*}{0.5} & 0.2 & 37.8550 & 19.4474 & 0.0010 & 0.9346 & 3.6179 & 375.1919 & 39.0991 & 32.4801 & 566.5620 \\
\hline & 0.5 & 36.4583 & 20.5938 & 0.0010 & 1.6021 & 5.0088 & 64.7990 & 44.6720 & 37.0097 & 573.6156 \\
\hline & 0.8 & 32.7976 & 22.2552 & 0.0010 & 1.2377 & 4.3834 & 360.7954 & 46.0753 & 37.4934 & 575.5708 \\
\hline \multirow[t]{3}{*}{0.8} & 0.2 & 29.2587 & 17.6455 & 0.0010 & 0.6277 & 2.8225 & 359.8273 & 48.1889 & 31.7879 & 582.6589 \\
\hline & 0.5 & 27.2532 & 17.4737 & 0.0010 & 0.8362 & 3.1991 & 367.9543 & 52.0129 & 35.1051 & 577.6879 \\
\hline & 0.8 & 25.9665 & 16.3588 & 0.0010 & 0.7279 & 3.1273 & 364.1244 & 50.0111 & 35.1252 & 573.5116 \\
\hline \multicolumn{2}{|c|}{ Total } & 36.4042 & 23.5445 & 0.0010 & 1.9700 & 5.9310 & 352.324 & 39.6631 & 35.7772 & 552.0780 \\
\hline
\end{tabular}

Fig. 5 describes the aggregated results considering $T F$. We can observe that matheuristic SPAM returned the best average results for the small-sized test instances. The MILP model obtained good results for $T F=2$ : however, for $T F$ equals 0.5 and 0.8 , it was outperformed by the SPAM and OMDD-S methods, respectively. Figure 6 illustrates the aggregated boxplots concerning the $R D D$ indicator. Considering these results, we can highlight that the SPAM matheuristic returned the better average results for all the methods under comparison.

Table 7

Small-sized test instances: \% of optimality for $T F$ and $R D D$

\begin{tabular}{|c|c|c|c|c|c|c|c|c|}
\hline$T F$ & $R D D$ & EDD & FP & FP-S & OMDD & OMDD-S & SPAM & MILP \\
\hline \multirow[t]{3}{*}{0.2} & 0.2 & $0,00 \%$ & $0,00 \%$ & $0,00 \%$ & $0,00 \%$ & $0,00 \%$ & $4,44 \%$ & $13,70 \%$ \\
\hline & 0.5 & $7,41 \%$ & $7,41 \%$ & $7,41 \%$ & $7,41 \%$ & $7,41 \%$ & $12,22 \%$ & $21,85 \%$ \\
\hline & 0.8 & $10,37 \%$ & $10,37 \%$ & $10,37 \%$ & $10,37 \%$ & $11,11 \%$ & $14,81 \%$ & $27,41 \%$ \\
\hline \multirow[t]{3}{*}{0.5} & 0.2 & $0,00 \%$ & $0,00 \%$ & $0,00 \%$ & $0,00 \%$ & $0,00 \%$ & $2,96 \%$ & $10,37 \%$ \\
\hline & 0.5 & $0,00 \%$ & $0,00 \%$ & $0,00 \%$ & $0,00 \%$ & $0,00 \%$ & $2,96 \%$ & $9,63 \%$ \\
\hline & 0.8 & $0,00 \%$ & $0,37 \%$ & $0,37 \%$ & $0,00 \%$ & $0,00 \%$ & $4,07 \%$ & $8,15 \%$ \\
\hline \multirow[t]{3}{*}{0.8} & 0.2 & $0,00 \%$ & $0,00 \%$ & $0,37 \%$ & $0,00 \%$ & $0,37 \%$ & $3,33 \%$ & $6,30 \%$ \\
\hline & 0.5 & $0,00 \%$ & $0,00 \%$ & $0,00 \%$ & $0,00 \%$ & $0,00 \%$ & $1,48 \%$ & $7,78 \%$ \\
\hline & 0.8 & $0,00 \%$ & $0,00 \%$ & $0,37 \%$ & $0,00 \%$ & $0,00 \%$ & $2,59 \%$ & $7,41 \%$ \\
\hline Total & & $1,98 \%$ & $2,02 \%$ & $2,10 \%$ & $1,98 \%$ & $2,10 \%$ & $5,43 \%$ & $12,51 \%$ \\
\hline
\end{tabular}

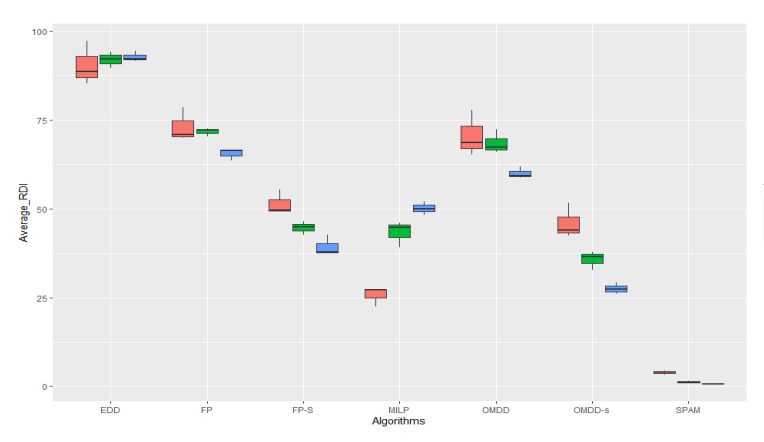

Fig. 5. Small-sized test instances: boxplot for average $R D I$ values depending on $T F$

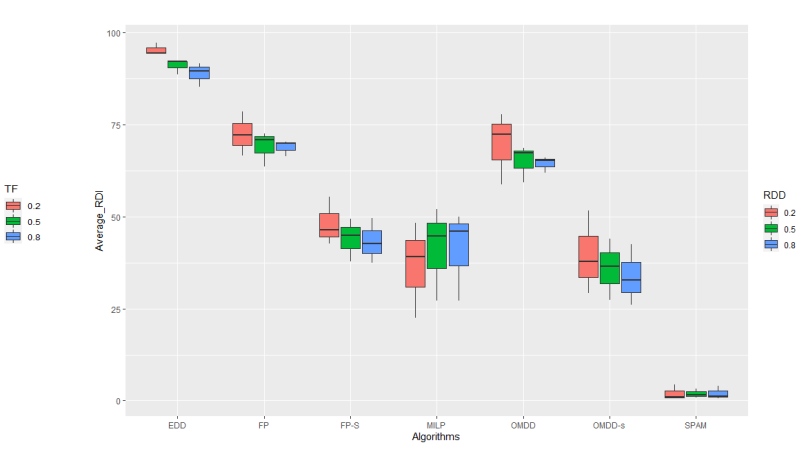

Fig. 6. Large-sized test instances: boxplot for average $R D I$ values depending on $R D D$

Table 8 presents the results for the $S R$ indicator. We can observe that the SPAM matheuristic returned the largest $S R$ value between all the evaluated methods. Based on the performance indicators analyzed in the computational results, we can conclude that SPAM matheuristic is the best method for the small-sized test instances.

Table 8

Small-sized test instances: SR values

\begin{tabular}{ccccccc}
\hline EDD & FP & FP-S & OMDD & OMDD-S & SPAM & MILP \\
\hline $1,98 \%$ & $0,65 \%$ & $0,93 \%$ & $0,82 \%$ & $1,37 \%$ & $30,75 \%$ & $8,93 \%$ \\
\hline
\end{tabular}

\subsubsection{Large-sized test instances}

Table 9 and Table 10 illustrates the computational results considering distinct values for $m$ and $n$. Taking into consideration the first set of instances $(m=2$ and $n=10)$, we can observe that the SPAM returns the best solutions among all the 
considered methods. However, with the increasing of the values of $m$ and $n$, MILP model and SPAM-JPO return the best $R D I$ values.

Table 9

Large-sized test instances: RDI values for $m$ and $n$ (part 1)

\begin{tabular}{ccccccccccccc}
\hline$m$ & $n$ & \multicolumn{2}{c}{ EDD } & \multicolumn{2}{c}{ FP } & \multicolumn{2}{c}{ FP-S } & \multicolumn{2}{c}{ OMDD } \\
& & Average & St. Dv. & Average & St. Dv. & Average & St. Dv. & Average & St. Dv. & Average & St. Dv. \\
\hline 2 & 10 & 96.0493 & 9.3616 & 74.0344 & 25.0666 & 56.2164 & 27.9392 & 71.7100 & 26.1257 & 38.9925 & 21.3457 \\
& 20 & 78.0808 & 39.7039 & 59.7296 & 36.7141 & 50.7682 & 35.8774 & 60.5183 & 37.2953 & 34.4581 & 28.589 \\
& 30 & 51.2699 & 47.9732 & 38.7929 & 38.8785 & 32.3783 & 34.8791 & 37.3421 & 38.5414 & 14.9562 & 21.4639 \\
& 40 & 30.6115 & 41.6665 & 20.7499 & 29.2799 & 16.2446 & 24.2014 & 20.6954 & 29.5093 & 5.5452 & 12.3045 \\
\hline 5 & 10 & 93.7887 & 12.8440 & 73.2340 & 23.6784 & 58.8424 & 26.2345 & 67.0770 & 26.035 & 37.4087 & 21.3143 \\
& 20 & 68.5931 & 38.5886 & 51.8989 & 33.9248 & 47.7679 & 33.6858 & 49.4103 & 34.2563 & 26.2327 & 27.6293 \\
& 30 & 24.8256 & 30.0024 & 15.7638 & 19.9920 & 14.0551 & 19.2174 & 14.7223 & 19.7352 & 5.0594 & 13.3444 \\
& 40 & 12.5403 & 18.9989 & 7.7343 & 11.3168 & 6.2689 & 9.0028 & 7.2335 & 12.0397 & 0.8205 & 3.8954 \\
\hline 8 & 10 & 91.5210 & 15.5189 & 68.1319 & 24.8727 & 56.2488 & 25.4111 & 64.4638 & 26.754 & 34.851 & 20.2135 \\
& 20 & 47.8643 & 34.6722 & 35.4441 & 30.6042 & 32.0611 & 30.927 & 34.0785 & 30.4616 & 16.3654 & 24.0684 \\
& 30 & 13.8092 & 17.0370 & 8.3775 & 9.3839 & 7.6681 & 9.6344 & 7.2338 & 9.0156 & 1.2870 \\
& 40 & 7.0822 & 10.0866 & 4.0645 & 5.4368 & 4.1008 & 5.6089 & 3.7166 & 5.1589 \\
\hline \multicolumn{2}{l}{ Total } & 51.3363 & 43.6517 & 38.1630 & 36.6161 & 31.8851 & 32.8421 & 36.5168 & 36.2386 & 18.0762 & 0.4868 \\
\hline
\end{tabular}

Table 10

Large-sized test instances: $R D I$ values for $m$ and $n$ (part 2)

\begin{tabular}{|c|c|c|c|c|c|c|c|c|c|}
\hline \multirow[t]{2}{*}{$m$} & \multirow[t]{2}{*}{$n$} & \multicolumn{2}{|c|}{ SPAM } & \multicolumn{2}{|c|}{ MILP } & \multicolumn{2}{|c|}{ SPAM-JPO } & \multicolumn{2}{|c|}{ JPO } \\
\hline & & Average & St. Dv. & Average & St. Dv. & Average & St. Dv. & Average & St. Dv. \\
\hline \multirow[t]{4}{*}{2} & 10 & 2.5691 & 5.0185 & 4.1034 & 6.0077 & 4.5762 & 6.4201 & 22.7252 & 18.4103 \\
\hline & 20 & 9.4939 & 13.0045 & 1.4788 & 3.8262 & 10.6221 & 15.5238 & 29.8185 & 25.6702 \\
\hline & 30 & 29.6135 & 35.5353 & 3.3109 & 11.3456 & 6.2066 & 9.5153 & 14.9408 & 21.4362 \\
\hline & 40 & 47.8850 & 46.3269 & 7.4285 & 16.0226 & 3.2077 & 7.8071 & 5.5452 & 12.3045 \\
\hline \multirow[t]{4}{*}{5} & 10 & 44.4169 & 31.2991 & 0.8070 & 3.4905 & 2.6394 & 5.1434 & 35.9667 & 20.1085 \\
\hline & 20 & 56.7089 & 40.8491 & 1.5417 & 5.5628 & 18.0544 & 24.0035 & 26.0185 & 27.2406 \\
\hline & 30 & 63.7955 & 47.0304 & 8.1344 & 18.8562 & 3.5100 & 10.4422 & 5.0594 & 13.3444 \\
\hline & 40 & 63.6526 & 48.1386 & 13.7993 & 23.4163 & 0.6903 & 3.8231 & 0.8205 & 3.8954 \\
\hline \multirow[t]{4}{*}{8} & 10 & 59.8336 & 29.7481 & 0.5792 & 2.9924 & 2.3233 & 5.0796 & 34.0763 & 19.5936 \\
\hline & 20 & 72.9362 & 42.2317 & 2.1991 & 6.2835 & 10.4192 & 18.2998 & 16.2987 & 23.8797 \\
\hline & 30 & 70.3357 & 45.7281 & 8.7350 & 15.6913 & 1.0305 & 2.7315 & 1.2870 & 3.4212 \\
\hline & 40 & 65.4765 & 47.5565 & 16.1070 & 24.7707 & 0.0684 & 0.4767 & 0.0762 & 0.4868 \\
\hline \multicolumn{2}{|c|}{ Total } & 48.8931 & 44.5045 & 5.6854 & 14.6673 & 5.2790 & 12.3564 & 16.0528 & 22.1632 \\
\hline
\end{tabular}

Table 11 describes the optimality percentages for the evaluated methods in the large-sized test instances, considering $m$ and $n$ values. We can observe that the MILP model and the hybrid matheuristic SPAM-JPO can find the largest number of optimal solutions. Analyzing the sets of instances separately, we can highlight that for $m=5$ and $n=10$, the MILP model obtained the largest percentage of optimality $(64.81 \%)$.

Table 11

Large-sized test instances: \% of optimality for $m$ and $n$

\begin{tabular}{|c|c|c|c|c|c|c|c|c|c|c|}
\hline$m$ & $n$ & EDD & FP & FP-S & OMDD & OMDD-S & SPAM & MILP & SPAM-JPO & JPO \\
\hline \multirow[t]{4}{*}{2} & 10 & $0.00 \%$ & $0.00 \%$ & $0.00 \%$ & $0.00 \%$ & $0.37 \%$ & $56.30 \%$ & $43.33 \%$ & $39.26 \%$ & $4.07 \%$ \\
\hline & 20 & $20.00 \%$ & $20.00 \%$ & $20.00 \%$ & $20.00 \%$ & $20.00 \%$ & $25.93 \%$ & $25.93 \%$ & $23.33 \%$ & $20.37 \%$ \\
\hline & 30 & $40.00 \%$ & $40.00 \%$ & $40.00 \%$ & $40.00 \%$ & $40.00 \%$ & $41.48 \%$ & $41.48 \%$ & $40.37 \%$ & $40.00 \%$ \\
\hline & 40 & $45.19 \%$ & $45.19 \%$ & $45.19 \%$ & $45.19 \%$ & $45.19 \%$ & $42.22 \%$ & $45.19 \%$ & $45.19 \%$ & $45.19 \%$ \\
\hline \multirow[t]{4}{*}{5} & 10 & $0.00 \%$ & $0.00 \%$ & $0.00 \%$ & $0.00 \%$ & $0.37 \%$ & $4.44 \%$ & $64.81 \%$ & $49.63 \%$ & $0.37 \%$ \\
\hline & 20 & $17.78 \%$ & $17.78 \%$ & 0.1778 & $17.78 \%$ & $17.78 \%$ & $20.74 \%$ & $21.11 \%$ & $17.78 \%$ & $17.78 \%$ \\
\hline & 30 & $33.70 \%$ & $33.70 \%$ & $33.70 \%$ & $33.70 \%$ & $33.70 \%$ & $33.33 \%$ & $34.07 \%$ & $33.70 \%$ & $33.70 \%$ \\
\hline & 40 & $40.37 \%$ & $40.37 \%$ & $40.37 \%$ & $40.37 \%$ & $40.37 \%$ & $35.93 \%$ & $40.37 \%$ & $40.37 \%$ & $40.37 \%$ \\
\hline \multirow[t]{4}{*}{8} & 10 & $0.00 \%$ & $0.00 \%$ & $0.00 \%$ & $0.00 \%$ & $0.37 \%$ & $0.37 \%$ & $55.19 \%$ & $40.74 \%$ & $0.37 \%$ \\
\hline & 20 & $15.56 \%$ & $15.56 \%$ & $15.56 \%$ & $15.56 \%$ & $15.56 \%$ & $19.26 \%$ & $19.26 \%$ & $16.30 \%$ & $15.56 \%$ \\
\hline & 30 & $31.85 \%$ & $31.85 \%$ & $31.85 \%$ & $31.85 \%$ & $31.85 \%$ & $28.89 \%$ & $31.85 \%$ & $31.85 \%$ & $31.85 \%$ \\
\hline & 40 & $38.15 \%$ & $38.15 \%$ & $38.15 \%$ & $38.15 \%$ & $38.15 \%$ & $33.33 \%$ & $38.15 \%$ & $38.15 \%$ & $38.15 \%$ \\
\hline \multicolumn{2}{|c|}{ Total } & $23.55 \%$ & $23.55 \%$ & $23.55 \%$ & $23.55 \%$ & $23.64 \%$ & $28.52 \%$ & $38.40 \%$ & $34.72 \%$ & $23.98 \%$ \\
\hline
\end{tabular}

Based on Table 9 and Table 10, we can observe that FP-S algorithm outperforms the FP heuristics since the first one presents smaller average $R D I$ values for all the sets of instances, except for the problems with $m=8$ and $n=40$. However, the difference between the RDI values between FP-S and FP heuristics in these test instances is only $0.0363 \%$. We can also emphisize that OMDD-S algorithm outperforms the OMDD heuristic since it returned best average $R D I$ values for all the test instances. Furthermore, OMDD-S presented the better results considering all the methods under comparison for five groups of test instances. 
Table 12 and Table 13 present the $R D I$ values (average and standard deviation) considering $T F$ and $R D D$. Once again, SPAM and OMDD-S have returned the best values for the RDI indicator. Also, the FP heuristic has presented the worst performance among the methods under comparison.

Table 12

Large-sized test instances: $R D I$ values for $T F$ and $R D D$ (part 1)

\begin{tabular}{|c|c|c|c|c|c|c|c|c|c|c|c|}
\hline \multirow[t]{2}{*}{$T F$} & \multirow[t]{2}{*}{$R D D$} & \multicolumn{2}{|c|}{ EDD } & \multicolumn{2}{|c|}{ FP } & \multicolumn{2}{|c|}{ FP-S } & \multicolumn{2}{|c|}{ OMDD } & \multicolumn{2}{|c|}{ OMDD-S } \\
\hline & & Average & St. Dv. & Average & St. Dv. & Average & St. Dv. & Average & St. Dv. & Average & St. Dv. \\
\hline \multirow[t]{3}{*}{0.2} & 0.2 & 49.7108 & 46.3151 & 37.9304 & 39.5661 & 32.3280 & 35.8786 & 39.0234 & 39.8507 & 24.8065 & 28.6791 \\
\hline & 0.5 & 44.1941 & 45.6207 & 35.7960 & 39.8416 & 33.3222 & 37.9785 & 35.1458 & 39.3492 & 21.5752 & 29.1973 \\
\hline & 0.8 & 35.8728 & 44.9106 & 27.9817 & 37.7023 & 24.3531 & 34.2296 & 26.2024 & 36.6533 & 14.4111 & 23.694 \\
\hline \multirow[t]{3}{*}{0.5} & 0.2 & 60.7937 & 42.2037 & 46.5010 & 38.2813 & 39.9098 & 35.5864 & 46.3644 & 37.7785 & 26.4463 & 26.4100 \\
\hline & 0.5 & 52.1433 & 44.0704 & 39.5498 & 37.8962 & 34.9246 & 35.7333 & 37.6886 & 37.8441 & 19.9796 & 26.1782 \\
\hline & 0.8 & 45.3101 & 44.6130 & 32.7084 & 35.6869 & 26.9918 & 31.5203 & 32.0222 & 36.1065 & 15.1196 & 22.7104 \\
\hline \multirow[t]{3}{*}{0.8} & 0.2 & 64.1586 & 36.8215 & 44.7169 & 30.9769 & 34.4809 & 25.7323 & 41.7298 & 30.6978 & 14.5048 & 17.2718 \\
\hline & 0.5 & 57.2549 & 38.8929 & 40.3317 & 31.0763 & 30.9592 & 25.4458 & 36.5535 & 30.5876 & 12.9058 & 16.5442 \\
\hline & 0.8 & 52.5883 & 41.7649 & 37.9509 & 34.1006 & 29.6960 & 28.5363 & 33.9210 & 32.5531 & 12.2908 & 17.1234 \\
\hline Total & & 51.3363 & 43.6517 & 38.1630 & 36.6161 & 31.8851 & 32.8421 & 36.5168 & 36.2386 & 18.0044 & 24.0698 \\
\hline
\end{tabular}

Table 13

Large-sized test instances: RDI values for $T F$ and $R D D$ (part 2)

\begin{tabular}{|c|c|c|c|c|c|c|c|c|c|}
\hline \multirow[t]{2}{*}{$T F$} & \multirow[t]{2}{*}{$R D D$} & \multicolumn{2}{|c|}{ SPAM } & \multicolumn{2}{|c|}{ MILP } & \multicolumn{2}{|c|}{ SPAM-JPO } & \multicolumn{2}{|c|}{ JPO } \\
\hline & & Average & St. Dv. & Average & St. Dv. & Average & St. Dv. & Average & St. Dv. \\
\hline \multirow[t]{3}{*}{0.2} & 0.2 & 22.0448 & 35.8186 & 2.5654 & 7.2692 & 6.6008 & 13.2974 & 20.9657 & 26.1605 \\
\hline & 0.5 & 25.3158 & 38.6867 & 3.9393 & 12.3742 & 5.7427 & 14.7685 & 19.8600 & 27.4597 \\
\hline & 0.8 & 26.1552 & 40.3818 & 5.5456 & 17.7981 & 3.3797 & 9.0318 & 12.6304 & 21.5056 \\
\hline \multirow[t]{3}{*}{0.5} & 0.2 & 39.4726 & 41.5005 & 1.9476 & 7.9712 & 9.1069 & 14.8537 & 23.5571 & 24.2580 \\
\hline & 0.5 & 45.1672 & 44.4587 & 2.9747 & 11.2436 & 7.7670 & 18.1712 & 17.6779 & 23.9755 \\
\hline & 0.8 & 58.6733 & 44.3084 & 5.8597 & 16.5763 & 4.5871 & 13.3385 & 14.0253 & 21.9911 \\
\hline \multirow[t]{3}{*}{0.8} & 0.2 & 71.9319 & 36.8566 & 7.7371 & 15.4082 & 3.6934 & 6.2072 & 12.9980 & 15.8555 \\
\hline & 0.5 & 75.3769 & 35.5378 & 9.9924 & 16.8006 & 3.1289 & 5.8179 & 11.6613 & 14.7730 \\
\hline & 0.8 & 75.9003 & 34.8084 & 10.6064 & 18.7741 & 3.5047 & 7.9139 & 11.0991 & 15.7275 \\
\hline \multicolumn{2}{|c|}{ Total } & 48.8931 & 44.5045 & 5.6854 & 14.6673 & 5.2790 & 12.3564 & 16.0528 & 22.1632 \\
\hline
\end{tabular}

Taking into consideration the Table 12 and 13, we can observe that FP-S algorithm outperforms the FP heuristic for all the evaluated test instances. In addition, the OMDD-S algorithm outperforms the OMMD heuristics also for all the test instances. Finally, we can highlight that the SPAM matheuristic presented better average RDI values than the MILP model for all the available test instances. Table 14 describes the percentage of optimality for the methods under comparison, classified by TF and RDD. We can observe that for $\mathrm{TF}=0.2$ and $\mathrm{RDD}=0.8$ the MILP model obtained the largest percentage of optimal solutions $(59.72 \%)$. Subsequently, the hybrid matheuristic SPAM-JPO can find a greater number of optimal solutions in comparison with the remainder methods.

Table 14

Large-sized test instances: \% of optimality for $T F$ and $R D D$

\begin{tabular}{|c|c|c|c|c|c|c|c|c|c|c|}
\hline$T F$ & $R D D$ & EDD & $\mathrm{FP}$ & FP-S & OMDD & OMDD-S & SPAM & MILP & SPAM-JPO & JPO \\
\hline \multirow[t]{3}{*}{0.2} & 0.2 & $41.67 \%$ & $41.67 \%$ & $41.67 \%$ & $41.67 \%$ & $41.67 \%$ & $46.39 \%$ & $51.11 \%$ & $50.28 \%$ & $41.94 \%$ \\
\hline & 0.5 & $44.44 \%$ & $44.44 \%$ & $44.44 \%$ & $44.44 \%$ & $44.44 \%$ & $56.39 \%$ & $56.39 \%$ & $53.06 \%$ & $45.00 \%$ \\
\hline & 0.8 & $49.72 \%$ & $49.72 \%$ & $49.72 \%$ & $49.72 \%$ & $49.72 \%$ & $57.78 \%$ & $59.72 \%$ & $58.06 \%$ & $50.28 \%$ \\
\hline \multirow[t]{3}{*}{0.5} & 0.2 & $25.00 \%$ & $25.00 \%$ & $25.00 \%$ & $25.00 \%$ & $25.00 \%$ & $30.56 \%$ & $38.33 \%$ & $34.44 \%$ & $25.28 \%$ \\
\hline & 0.5 & $26.11 \%$ & $26.11 \%$ & $26.11 \%$ & $26.11 \%$ & $26.11 \%$ & $32.22 \%$ & $42.78 \%$ & $36.67 \%$ & $26.67 \%$ \\
\hline & 0.8 & $24.17 \%$ & $24.17 \%$ & $24.17 \%$ & $24.17 \%$ & $24.44 \%$ & $20.56 \%$ & $42.78 \%$ & $38.33 \%$ & $25.00 \%$ \\
\hline \multirow[t]{3}{*}{0.8} & 0.2 & $0.00 \%$ & $0.00 \%$ & $0.00 \%$ & $0.00 \%$ & $0.00 \%$ & $4.17 \%$ & $18.06 \%$ & $13.89 \%$ & $0.00 \%$ \\
\hline & 0.5 & $0.00 \%$ & $0.00 \%$ & $0.00 \%$ & $0.00 \%$ & $0.56 \%$ & $4.44 \%$ & $17.78 \%$ & $13.06 \%$ & $0.83 \%$ \\
\hline & 0.8 & $0.83 \%$ & $0.83 \%$ & $0.83 \%$ & $0.83 \%$ & $0.83 \%$ & $4.17 \%$ & $18.61 \%$ & $14.72 \%$ & $0.83 \%$ \\
\hline \multicolumn{2}{|c|}{ Total } & $23.55 \%$ & $23.55 \%$ & $23.55 \%$ & $23.55 \%$ & $23.64 \%$ & $28.52 \%$ & $38.40 \%$ & $34.72 \%$ & $23.98 \%$ \\
\hline
\end{tabular}

Fig. 7 illustrates the boxplot for average $R D I$ values taking into consideration the parameter $T F$. Given the obtained results, we can highlight that the JPO-SPAM matheuristic returned the best results for all the values of $T F$. Besides, the MILP model presented the worse results for $T F$ equals to 0.8 . Figure 8 illustrates the average $R D I$ values depending on $R D D$. Once again, the hybrid matheuristic SPAM-JPO presented better results than all the other methods under comparison. Table 15 presents the SR indicator for each evaluated method. We can observe that the MILP model can find the largest number of best solutions. In fact, for the large-sized instances, the difference between the MILP model and the hybrid matheuristic SPAM-JPO is not statistically significant.

Table 15

Large-sized test instances: SR values

\begin{tabular}{ccccccccc}
\hline EDD & FP & FP-S & OMDD & OMDD-S & SPAM & MILP & SPAM-JPO & JPO \\
\hline $28.43 \%$ & $28.43 \%$ & $28.52 \%$ & $28.43 \%$ & $43.95 \%$ & $32.47 \%$ & $69.32 \%$ & $59.81 \%$ & $44.29 \%$ \\
\hline
\end{tabular}




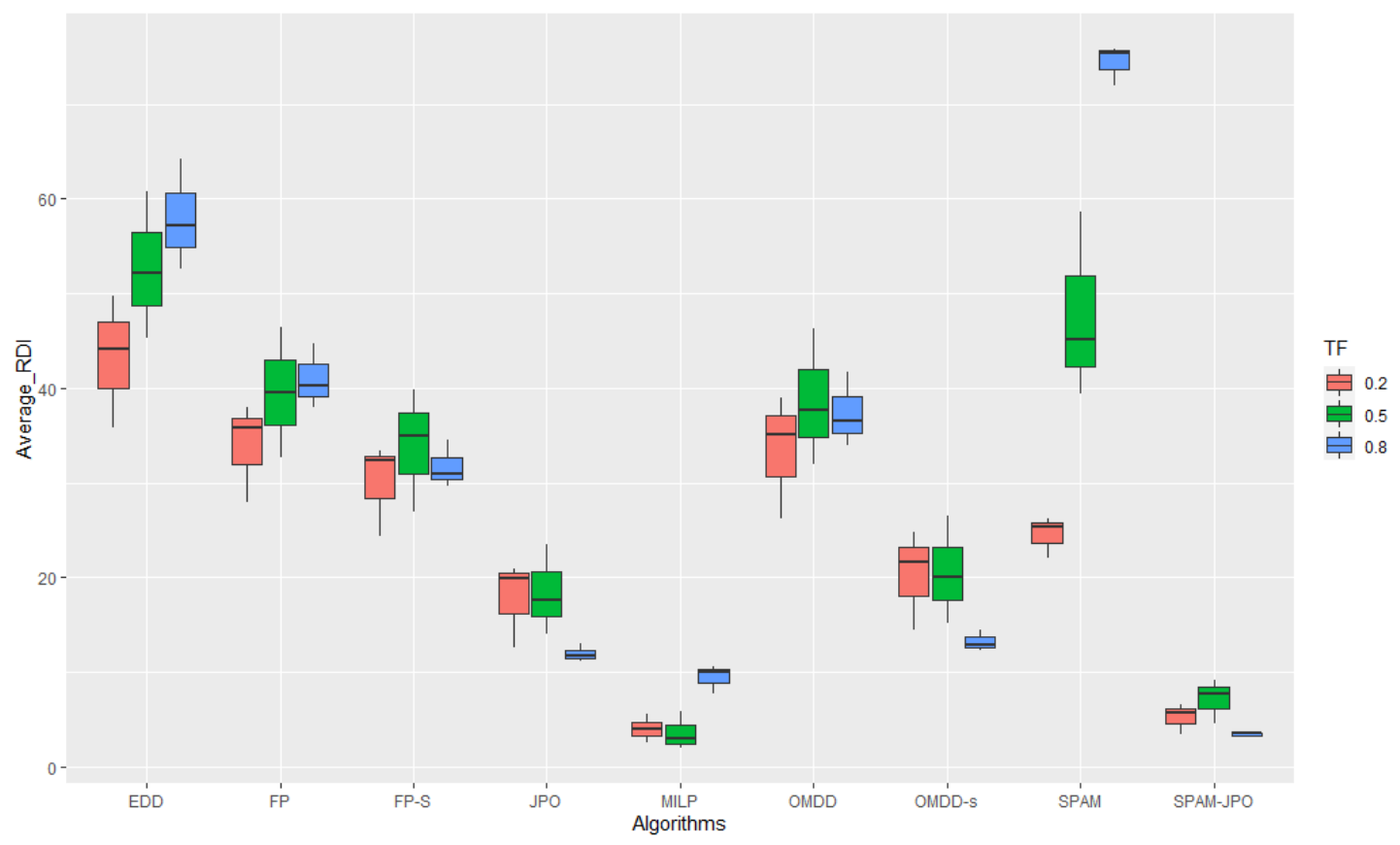

Fig. 7. Large-sized test instances: boxplot for average $R D I$ values depending on $T F$

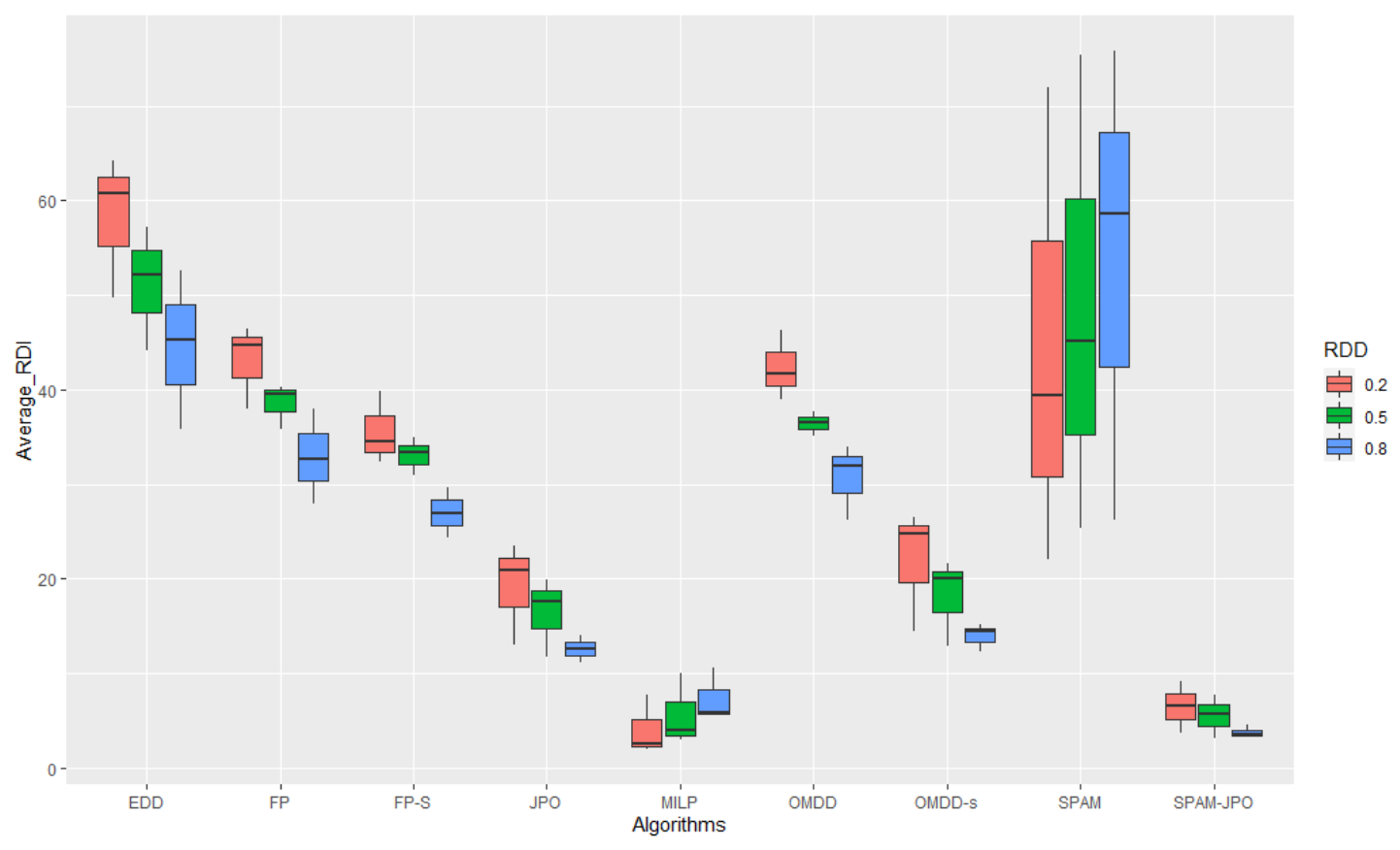

Fig. 8. Large-sized instances: boxplot for average $R D I$ values depending on $R D D$

\section{Conclusions}

This paper has introduced a COSP that considers sequence-dependent setup time for the total completion time minimization. An MILP model has been proposed for the proposed variant. Besides, two constructive heuristics, as well as two matheuristics, have also been presented. Extensive computational experiments have been reported with two datasets composed of randomly generated test instances, aiming to evaluate the methods under comparison. As the performance indicators, the study used the $\mathrm{RDI}$, the SR, and the average computational time. We evaluated 5670 instances, divided into 2430 small-sized and 3240 largesized instances. Based on the computational experience, we can observe that for the small-sized test instances, the SPAM matheuristic and the OMDD-S constructive heuristic returned the best average RDI values. Thus, our proposal presented better results than all the other methods under comparison. Concerning the large-sized test instances, the proposed the hybrid 
matheuristic SPAM-JPO outperforms the JPO metaheuristic - which is the state-of-the-art solution method for the COSP to minimize total tardiness - with the same time limit. Therefore, the proposed matheuristic is a promising method to solve the problem under study. As suggestions for future studies, other matheuristics and matheuristics could be developed. The OMDD-S heuristic could be used as a warm start to MILP and SPAM methods, improving the solutions returned by these methods. Another research avenue is the consideration of other performance measures, such as total earliness or just-in-time problems.

\section{Acknowledgments}

This study was financed in part by the Coordination for the Improvement of Higher Education Personnel (CAPES), the National Council for Scientific and Technological Development (CNPq), through Grant no. 303595/2018-7.

\section{References}

Fernandez-Viagas, V., \& Framinan, J.M. (2015). Neh-based heuristics for the permutation flowshop scheduling problem to minimize total tardiness. Computers and Operations Research, 60, 27-36.

Fernandez-Viagas, V., Perez-Gonzalez, P., \& Framinan, J. M. (2019). Efficiency of the solution representations for the hybrid flow shop scheduling problem with makespan objective. Computers \& Operations Research, 109, 77-88.

Framinan, J.M., \& Perez-Gonzalez, P. (2017). New approximate algorithms for the customer order scheduling problem with total completion time objective. Computers and Operations Research, 78, $181-192$.

Framinan, J.M., \& Perez-Gonzalez, P. (2018). Order scheduling with tardiness objective: Improved approximate solutions. European Journal of Operational Research, 266(3), 840 - 850.

Framinan, J.M., Perez-Gonzalez, P., \& Fernandez-Viagas, V. (2019). Deterministic assembly scheduling problems: A review and classification of concurrent-type scheduling models and solution procedures. European Journal of Operational Research, 273(2), $401-417$.

Karabulut, K. (2016). A hybrid iterated greedy algorithm for total tardiness minimization in permutation flowshops. Computers and Industrial Engineering, 98, 300 - 307.

Kim, Y.D. (1993). Heuristics for flowshop scheduling problems minimizing mean tardiness. Journal of the Operational Research Society, 44(1), 19-28.

Kung, J.Y., Duan, J., Xu, J., Chung, I., Cheng, S.R., Wu, C.C., Lin, W.C., et al. (2018). Metaheuristics for order scheduling problem with unequal ready times. Discrete Dynamics in Nature and Society.

Lee, I.S. (2013). Minimizing total tardiness for the order scheduling problem. International Journal of Production Economics, 144(1), $128-134$.

Leung, J.Y.T., Li, H., \& Pinedo, M. (2005a). Order scheduling models: An overview. Multidisciplinary Scheduling: Theory and Applications, Springer US, Boston, MA., 37-53.

Leung, J.Y.T., Li, H., \& Pinedo, M. (2005b). Order scheduling in an environment with dedicated re-sources in parallel. Journal of Scheduling, 8(5), 355-386.

Leung, J.Y.T., Li, H., \& Pinedo, M. (2006). Scheduling orders for multiple product types with duedate related objectives. European Journal of Operational Research, 168(2), 370 - 389.

Lin, W.C., Xu, J., Bai, D., Chung, I.H., Liu, S.C., \& Wu, C.C. (2019). Artificial bee colony algorithmsfor the order scheduling with release dates. Soft Computing, 23(18), 8677-8688.

Michael, L.P. (2018). Scheduling: theory, algorithms, and systems. Springer.

Prata, B.A., Rodrigues, C.D., \& Framinan, J.M. (2021a). Customer order scheduling problem to minimize makespan with sequence-dependent setup times. Computers \& Industrial Engineering, 151, 106962.

Prata, B.A., Rodrigues, C.D., \& Framinan, J.M. (2021b). A differential evolution algorithm for the customer order scheduling problem with sequence-dependent setup times. Expert Systems With Applications, 116097.

Riahi, V., Newton, M.H., Polash, M., \& Sattar, A. (2019). Tailoring customer order scheduling search algorithms. Computers \& Operations Research, 108, $155-165$.

Roemer, T.A. (2006). A note on the complexity of the concurrent open shop problem. Journal of Scheduling, 9(4), 389-396.

Wagneur, E., Sriskandarajah, C. (1993). Openshops with jobs overlap. European Journal of Operational Research, 71(3), $366-378$.

Wu, C.C., Yang, T.H., Zhang, X., Kang, C.C., Chung, I.H., Lin, W.C. (2019). Using heuristic and iterative greedy algorithms for the total weighted completion time order scheduling with release times. Swarm and Evolutionary Computation, 44, $913-926$.

Wu, C. C., Bai, D., Zhang, X., Cheng, S. R., Lin, J. C., Wu, Z. L., \& Lin, W. C. (2021). A robust customer order scheduling problem along with scenario-dependent component processing times and due dates. Journal of Manufacturing Systems, 58, 291-305.

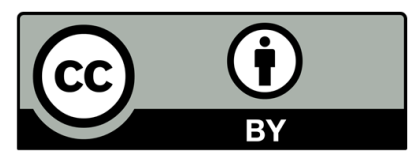

(C) 2022 by the authors; licensee Growing Science, Canada. This is an open access article distributed under the terms and conditions of the Creative Commons Attribution (CCBY) license (http://creativecommons.org/licenses/by/4.0/). 